\title{
Impact of plasma potassium normalization on short-term mortality in patients with hypertension and hypokalemia or low normal potassium
}

Maria Lukács Krogager ${ }^{1 *}$ (D) Peter Søgaard ${ }^{1}$, Christian Torp-Pedersen², Henrik Bøggild ${ }^{3,4}$, Christina Ji-Young Lee ${ }^{2,5}$, Anders Bonde ${ }^{5}$, Jesper Q. Thomassen ${ }^{6}$, Gunnar Gislason ${ }^{7,8,9}$, Manan Pareek ${ }^{2,10,11}$ and Kristian Kragholm 1,4,12

\begin{abstract}
Background: Hypokalemia is common in patients treated with antihypertensive drugs, but the impact of correcting hypokalemia is insufficiently studied. We examined the consequences of hypokalemia and borderline hypokalemia correction in patients with hypertension.

Methods: We identified 8976 patients with hypertension and plasma potassium concentrations $\leq 3.7 \mathrm{mmol} / \mathrm{L}$ within 100 days from combination antihypertensive therapy initiation. The first measurement between 6 and 100 days after the episode with potassium $\leq 3.7 \mathrm{mmol} / \mathrm{L}$ was retained. We investigated all-cause and cardiovascular mortality within 60-days from the second potassium measurement using Cox regression. Mortality was examined for seven predefined potassium intervals derived from the second measurement: $1.5-2.9 \mathrm{mmol} / \mathrm{L}(n=271), 3.0-3.4 \mathrm{mmol} / \mathrm{L}$ $(n=1341), 3.5-3.7(n=1982) \mathrm{mmol} / \mathrm{L}, 3.8-4.0 \mathrm{mmol} / \mathrm{L}(n=2398$, reference), $4.1-4.6 \mathrm{mmol} / \mathrm{L}(n=2498), 4.7-5.0 \mathrm{mmol} /$ $\mathrm{L}(n=352)$ and $5.1-7.1 \mathrm{mmol} / \mathrm{L}(n=134)$.

Results: Multivariable analysis showed that potassium concentrations $1.5-2.9 \mathrm{mmol} / \mathrm{L}, 3.0-3.4 \mathrm{mmol} / \mathrm{L}, 4.7-5.0$ $\mathrm{mmol} / \mathrm{L}$ and $5.1-7.1 \mathrm{mmol} / \mathrm{L}$ were associated with increased all-cause mortality (HR 2.39, 95\% Cl 1.66-3.43; HR 1.36, 95\% Cl 1.04-1.78; HR 2.36, 95\% Cl 1.68-3.30 and HR 2.62, 95\% Cl 1.73-3.98, respectively). Potassium levels $<3.0$ and $>4.6 \mathrm{mmol} / \mathrm{L}$ were associated with increased cardiovascular mortality. The adjusted standardized 60-day mortality risks in the seven strata were: $11.7 \%$ (95\% Cl 8.3-15.0\%), 7.1\% (95\% Cl 5.8-8.5\%), 6.4\% (95\% Cl 5.3-7.5\%), 5.4\% (4.5-6.3\%), 6.3\% (5.4-7.2\%), 11.6\% (95\% Cl 8.7-14.6\%) and 12.6\% (95\% Cl 8.2-16.9\%), respectively.

Conclusions: Persistent hypokalemia was frequent and associated with increased all-cause and cardiovascular mortality. Increase in potassium to levels $>4.6 \mathrm{mmol} / \mathrm{L}$ in patients with initial hypokalemia or low normal potassium was associated with increased all-cause and cardiovascular mortality.
\end{abstract}

Keywords: Hypokalemia, Borderline hypokalemia, Hypokalemia correction, Mortality, Low potassium.

\footnotetext{
* Correspondence: Ikcsmaria@yahoo.com; maria.krogager@rn.dk

'Department of Cardiology, Aalborg University Hospital, Aalborg, Denmark

Full list of author information is available at the end of the article
}

(c) The Author(s). 2020 Open Access This article is licensed under a Creative Commons Attribution 4.0 International License, which permits use, sharing, adaptation, distribution and reproduction in any medium or format, as long as you give appropriate credit to the original author(s) and the source, provide a link to the Creative Commons licence, and indicate if changes were made. The images or other third party material in this article are included in the article's Creative Commons licence, unless indicated otherwise in a credit line to the material. If material is not included in the article's Creative Commons licence and your intended use is not permitted by statutory regulation or exceeds the permitted use, you will need to obtain permission directly from the copyright holder. To view a copy of this licence, visit http://creativecommons.org/licenses/by/4.0/ The Creative Commons Public Domain Dedication waiver (http://creativecommons.org/publicdomain/zero/1.0/) applies to the data made available in this article, unless otherwise stated in a credit line to the data. 


\section{Novelty and Significance}

What is new?

- $\quad$ Correcting plasma potassium concentrations $\leq 3.7$ $\mathrm{mmol} / \mathrm{L}$ to levels between $3.5-4.6 \mathrm{mmol} / \mathrm{L}$ was associated with improved short-term prognosis

What is relevant?

- Increased mortality risk was observed in patients who initially had borderline hypokalemia, partly because they developed hypokalemia. This emphasizes that potassium supplementation might be relevant in patients with low normal potassium concentrations

- Correcting hypokalemia and borderline hypokalemia shortly was associated with good prognosis

- Low potassium concentrations have previously been associated with arrhythmogenesis and increased mortality risk in patients with hypertension.

\section{Summary}

In this register based study we investigated the impact of correcting hypokalemia and borderline hypokalemia on 60-day mortality among 8976 patients treated with combination antihypertensive therapy. We observed that: (1) persistent hypokalemia was common and associated with increased all-cause and cardiovascular mortality; (2) Increase in potassium to levels $>4.6 \mathrm{mmol} / \mathrm{L}$ in patients with initial hypokalemia or low normal potassium was associated with increased all-cause and cardiovascular mortality; (3) Among patients with borderline hypokalemia initially, development of hypokalemia or hyperkalemia was associated with increased mortality risk; (4) Correcting hypokalemia associated with increased survival.

\section{Background}

Several common clinical conditions and drugs are known to cause or precipitate hypokalemia [1]. Among patients with hypertension, thiazides are the antihypertensive drugs most frequently associated with hypokalemia [2-4].

We recently demonstrated a U-shaped relationship between potassium levels and mortality among patients with hypertension. We observed an increased mortality risk even in patients with low and high normal serum potassium concentrations, suggesting a narrower than previously thought normal interval for potassium of 4.1$4.7 \mathrm{mmol} / \mathrm{L}$. [5] However, at present there is no evidence regarding the consequences of potassium normalization in patients with hypertension and hypokalemia. Therefore, it is essential to examine how correction and even overcorrection of hypokalemia affect prognosis in patients with hypertension.

Using Danish national registers, we investigated the 60-day mortality among patients with hypertension and hypokalemia or low normal potassium concentrations, according to their subsequent plasma potassium concentrations measured within 6-100 days following the initial episode with low potassium levels.

\section{Methods}

\section{Data sources}

In Denmark, a unique and personal identification number is allocated to all individuals at the time of birth or immigration. This unique identifier allows linkage of health and administrative data at the individual level [6] and ensures nearly complete follow-up. We used anonymized data from five different registers made available by Statistics Denmark after central encryption of the unique identifiers [7]. An overview of the registers used in this study is available in Supplementary Table S1. In Denmark, register-based studies using anonymized data provided by Statistics Denmark are not warranted approval from the ethics committee.

\section{Study population}

We defined hypertension as redemption of minimum two antihypertensive agents in two consecutive quarters. This definition has previously been validated [8]. Patients entered the present study in the second quarter, referred to as the date of hypertension. An overview of the Anatomical Therapeutic Chemical Classification System (ATC) codes used to identify patients with hypertension is available in Supplementary Table S2. We required a plasma potassium measurement $\leq 3.7 \mathrm{mmol} / \mathrm{L}$ within 100 days from the date of hypertension for inclusion. The first measurement within this time interval was retained and referred to as the first potassium measurement $\left(\mathrm{K}_{1}\right)$. The second potassium measurement $\left(\mathrm{K}_{2}\right)$ was identified in the interval 6-100 days from $K_{1}$ and the first draw within this timeframe was retained. We did not include potassium concentrations within 1-5 days from $K_{1}$ as, potassium disarrays are usually corrected within a few days, regardless of the strategies applied. Patients below 18 years of age were excluded from the study. Supplementary Figure S1 illustrates the population flowchart.

\section{Comorbidities and medication}

We identified comorbidities and medications regarded as confounders when studying the association between changes in potassium levels and short-term mortality. The following comorbidities dated up to 5 years before the index date $\left(\mathrm{K}_{2}\right.$ date) were identified: hospitalization for heart failure, ischemic heart disease, stroke, chronic 
obstructive pulmonary disease, chronic liver disease, diabetes mellitus, inflammatory bowel disease and malignancy. Furthermore, patients with a past history of primary adrenal insufficiency, primary hyperaldosteronism, and diabetes insipidus were excluded. The International Classification of Disease (ICD) codes used to identify above-mentioned comorbidities are shown in Supplementary Table S3. We used the Chronic Kidney Disease Epidemiology Collaboration (CKD-EPI) equation [9] to calculate renal function, and an estimated glomerular filtration rate $($ eGFR $)<30 \mathrm{~mL} / \mathrm{min} / 1.73 \mathrm{~m}^{2}$ described significant renal insufficiency. Patients were excluded if no creatinine concentrations were available the same day as or within a week from the index date. Patients with missing serum sodium measurements on the index date were also excluded.

Prescriptions redeemed up to 90-days before the index date were identified for the following drugs: potassium supplements, non-steroidal anti-inflammatory drugs, corticosteroids, laxatives, xanthines, and antimicrobials. See Supplementary Table S3 for relevant ATC codes.

\section{Exposure variable}

Serum and plasma measurements yield similar results, but for serum samples there is a risk of contamination with potassium from burst platelets during coagulation in the range of $0.1-0.5 \mathrm{mmol} / \mathrm{L}$ due to non-standard sample handling [10]. Therefore, we only used plasma potassium measurements.

There is not a consensus on the normal plasma potassium interval, as it can vary from population to population. Supplementary Table S4 gives an overview on the three most used reference intervals in serum and plasma originating from different populations. We defined hypokalemia as plasma potassium concentrations below 3.5 $\mathrm{mmol} / \mathrm{L}$ and borderline hypokalemia as potassium levels within the interval $3.5-3.7 \mathrm{mmol} / \mathrm{L}$. Hyperkalemia was defined as potassium levels above $4.6 \mathrm{mmol} / \mathrm{L}$. [11] For $\mathrm{K}_{2}$, seven predefined potassium intervals were constructed: $1.5-2.9 \mathrm{mmol} / \mathrm{L}, \quad 3.0-3.4 \mathrm{mmol} / \mathrm{L}, 3.5-3.7$ $\mathrm{mmol} / \mathrm{L}, \quad 3.8-4.0 \mathrm{mmol} / \mathrm{L}, \quad 4.1-4.6 \mathrm{mmol} / \mathrm{L}, \quad 4.7-5.0$ $\mathrm{mmol} / \mathrm{L}$ and $5.1-7.1 \mathrm{mmol} / \mathrm{L}$. Plasma potassium interval $\mathrm{K}: 3.8-4.0 \mathrm{mmol} / \mathrm{L}$ was used as the reference for statistical analyses. We chose this interval as the reference group because it had one of the largest number of patients and lowest mortality rate.

\section{Outcome}

The primary outcome was all-cause mortality within 60 days from $K_{2}$. The secondary outcome was presumed cardiovascular death within 60 days from $\mathrm{K}_{2}$.

\section{Statistical analyses}

Categorical variables were presented as counts and percentages, and continuous variables as median with corresponding 25th and 75th percentiles. Differences between variables were compared using chi-squared and KruskalWallis tests, as appropriate.

To illustrate survival probability, Kaplan Meier curves were plotted for the seven potassium intervals. A restricted cubic spline curve was constructed to investigate the relationship between potassium as a continuous variable and absolute mortality risk in an age, sex, comorbidity and drug standardized population.

Cox proportional hazard modeling was used to analyze the association between the seven predefined potassium intervals and 60-day all-cause and presumed cardiovascular mortality. Based on the Cox regression principle, we modelled an average effect to estimate the 60-day absolute risk of all-cause mortality, with potassium interval $3.8-4.0 \mathrm{mmol} / \mathrm{L}$ as reference.

The multivariable model was adjusted for: age, sex, serum sodium, renal insufficiency, malignancy, heart failure, chronic liver disease, chronic obstructive pulmonary disease, diabetes mellitus, stroke, atrial flutter/fibrillation, ischemic heart disease, inflammatory bowel disease, antihypertensive therapy, corticosteroids, antimicrobials, non-steroidal anti-inflammatory drugs, xanthines, laxatives, and potassium supplements. The proportional hazard assumption was tested by plotting Schoenfeld residuals and was not violated. Interactions on mortality were tested by comparing the likelihood ratio of the Cox regression model with and without the interaction term. The following variables were tested for interaction with plasma potassium on mortality: age, sex, and renal insufficiency. A two-sided $p$-value $<0.01$ was considered statistically significant for interactions. We found no statistically significant interactions. For other analyses, a two-sided $p$-value $<0.05$ was considered statistically significant. Linearity of age on mortality was also assessed through a likelihood ratio test comparing a linear description with a categorical one. Age was found to violate linearity and was included as a categorical variable with five levels, using cut-off values from every 20th percentiles (55, 64, 72, 79 and 101 years). Hazard ratios (HR) and absolute risks (AR) were estimated with 95\% confidence intervals (95\% CI). All data management and analyses were performed using SAS, version 9.4 and $\mathrm{R}$, version 3.5.0 [12].

\section{Results}

\section{Demographics}

We identified 8976 patients treated with combination antihypertensive therapy who had potassium concentrations $\leq 3.7 \mathrm{mmol} / \mathrm{L}$ within the first 100 days from combination therapy initiation. Baseline characteristics for 
the cohort stratified on the seven predefined $K_{2}$ intervals are presented in Table 1 . Females accounted for $53 \%$ of the total population and median age was 68.3 years (range 18.2-100.8 years). Of the patients with borderline hypokalemia at $\mathrm{K}_{1}, 13 \%$ developed hypokalemia and $5.7 \%$ hyperkalemia at $K_{2}$. As for patients with hypokalemia at $\mathrm{K}_{1}$, we observed that $28.5 \%$ remained hypokalemic at the second blood draw and 4.8\% developed hyperkalemia. Approximately half of the population was hospitalized at $K_{1}$ and four fifths at $K_{2}$. See supplementary Figure S2 displaying the distribution of $K_{1}$, average of potassium measurements drawn within 1-5 days from $K_{1}$, and $K_{2}$. A low number of patients $(n=572)$ had renal insufficiency at the time of second potassium draw. Median time from $K_{1}$ to $K_{2}$ was 22 days (range: 6-100 days). As for diuretic treatment, thiazides were common in patients with potassium concentrations $\leq 3.7 \mathrm{mmol} / \mathrm{L}$, whereas loop diuretics were more common among patients with high potassium levels. Thiazide-like diuretics accounted for $4.4 \%$ of the total prescriptions of thiazides.

Demographics stratified on survival status showed that age, renal insufficiency, lower sodium concentrations, hospitalization at the time $K_{1}$, prior history of malignancy, chronic liver disease, chronic obstructive pulmonary disease, atrial fibrillation/atrial flutter, heart failure, and stroke were predominant among the deceased (Supplementary Table S5).

60-day survival after the second potassium measurement During 60-day follow-up after $K_{2}, 627$ (7.0\%) patients died, 331 from a cardiovascular cause. Mortality in the seven strata was: $14.4,7.0,6.3,5.2,6.7,13.6$ and $21.6 \%$, respectively. The restricted cubic spline curve revealed a U-shaped relationship between potassium and mortality (Fig. 1).

The results of the multivariable Cox regression, with plasma potassium $3.8-4.0 \mathrm{mmol} / \mathrm{L}$ as the reference group are shown in Fig. 2. All-cause mortality was significantly increased in patients with hypokalemia (1.5$2.9 \mathrm{mmol} / \mathrm{L}$ HR $2.39,95 \%$ CI $1.66-3.43$ and $3.0-3.4$ $\mathrm{mmol} / \mathrm{L}$ HR 1.36, 95\% CI 1.04-1.78) when compared with the reference. We observed a trend towards increased mortality in patients with borderline hypokalemia and with potassium levels within the interval 4.1$4.6 \mathrm{mmol} / \mathrm{L}$ (HR 1.24, 95\% CI 0.97-1.59 and HR 1.20, 95\% CI 0.95-1.51, respectively). All-cause mortality was also elevated in patients with hyperkalemia (4.7-5.0 $\mathrm{mmol} / \mathrm{L} \mathrm{HR} 2.36,95 \%$ CI 1.68-3.30; 5.1-5.7 mmol/L HR 2.62, 95\% CI 1.73-3.98). The univariable analysis showed similar results. We observed no interaction between $K_{1}$ and $\mathrm{K}_{2}$ on 60 -day mortality.

Cardiovascular mortality accounted for nearly 53\% of all deaths. We observed increased risk of cardiovascular death in patients with initial hypokalemia or low normal potassium levels who had potassium concentrations < $3.0 \mathrm{mmol} / \mathrm{L}$ and $>4.6 \mathrm{mmol} / \mathrm{L}$ at the second measurement.

The standardized 60-day absolute risk of all-cause mortality was lowest in patients with potassium concentrations between $3.8-4.0 \mathrm{mmol} / \mathrm{L}$ (AR 5.4, 95\% CI 4.5$6.3 \%$, Table 2). Significant differences in risks (reported against the reference) were observed for the following potassium intervals: $1.5-2.9 \mathrm{mmol} / \mathrm{L}$ risk difference $6.3 \%$ (95\% CI 2.9-9.7\%); 4.7-5.0 mmol/L risk difference $6.2 \%$ (95\% CI 3.2-9.3\%); 5.1-7.1 mmol/L risk difference $7.2 \%$ (95\% CI 2.8-11.6\%).

\section{Subgroup and sensitivity analyses}

We performed eleven additional analyses to test the accuracy and robustness of the main results (Table S6).

First, multivariable analysis performed on a subgroup of patients without kidney insufficiency showed that potassium levels within the intervals $1.5-2.9 \mathrm{mmol} / \mathrm{L}$ and $3.0-3.4 \mathrm{mmol} / \mathrm{L}$ were associated with increased mortality risk compared with the reference $(3.8-4.0 \mathrm{mmol} / \mathrm{L})$ (HR 2.33, 95\% CI $1.56-3.46$ and HR $1.35,95 \%$ CI $1.02-1.79$, respectively).

Second, in a subpopulation without history of malignancy, adjusted analyses showed that potassium concentrations outside the interval $3.0-4.6 \mathrm{mmol} / \mathrm{L}$ were associated with increased risk of death compared with the reference.

Third, subgroup analysis on patients without history of heart failure and no loop diuretic prescription showed that patients with hypokalemia and hyperkalemia had an increased mortality risk compared with patients with potassium levels in the interval $3.8-4.0 \mathrm{mmol} / \mathrm{L}$.

Fourth, analysis performed on a subgroup of patients without ischemic heart disease showed that patients with severe hypokalemia, and hyperkalemia had increased risk short-term mortality risk when compared with the reference.

Fifth, looking at patients with borderline hypokalemia at the first potassium measurement, we observed that patients who developed hypokalemia $(1.5-2.9 \mathrm{mmol} / \mathrm{L}$ : HR 2.16, 95\% CI 1.25-3.73; 3.0-3.4 mmol/L: HR 1.70, 95\% CI $1.22-2.37)$, or hyperkalemia $(4.7-5.0 \mathrm{mmol} / \mathrm{L}$ : HR 1.84, 95\% CI 1.18-2.86; 5.1-7.1 mmol/L: HR 2.81, 95\% CI 1.68-4.71) had an increased risk of death within 60-days when compared with the reference.

Sixth, among patients with hypokalemia at $K_{1}$, analyses showed that potassium concentrations within the intervals $1.5-2.9 \mathrm{mmol} / \mathrm{L}, 4.1-4.6 \mathrm{mmol} / \mathrm{L}$ and $4.7-5.0 \mathrm{mmol} /$ $\mathrm{L}$ were associated with increased short-term mortality risk.

Seventh, by performing the analyses on the last available potassium measurement within $6-100$ days from $K_{1}$ 


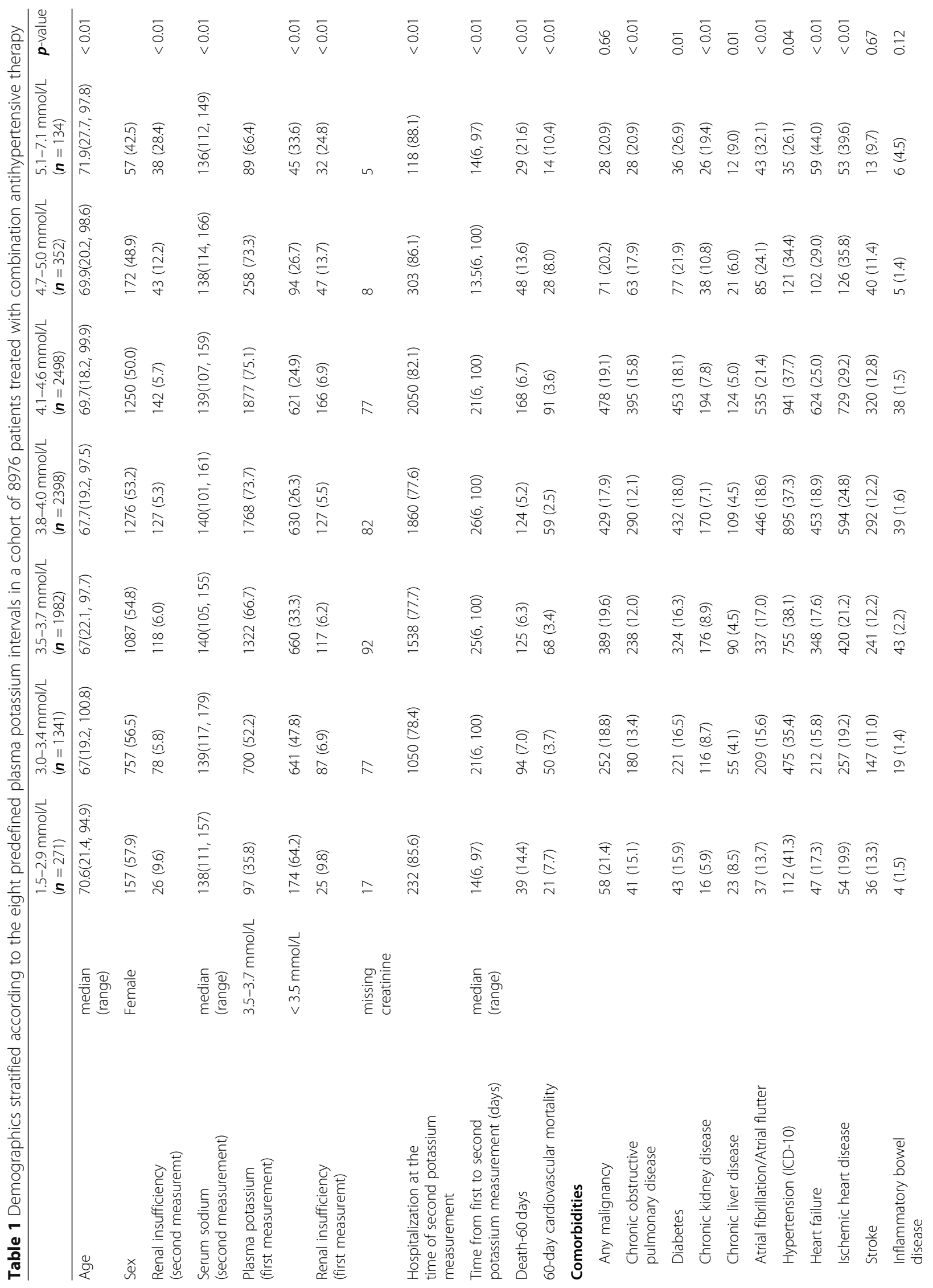


Krogager et al. BMC Cardiovascular Disorders

(2020) 20:386

Page 6 of 11

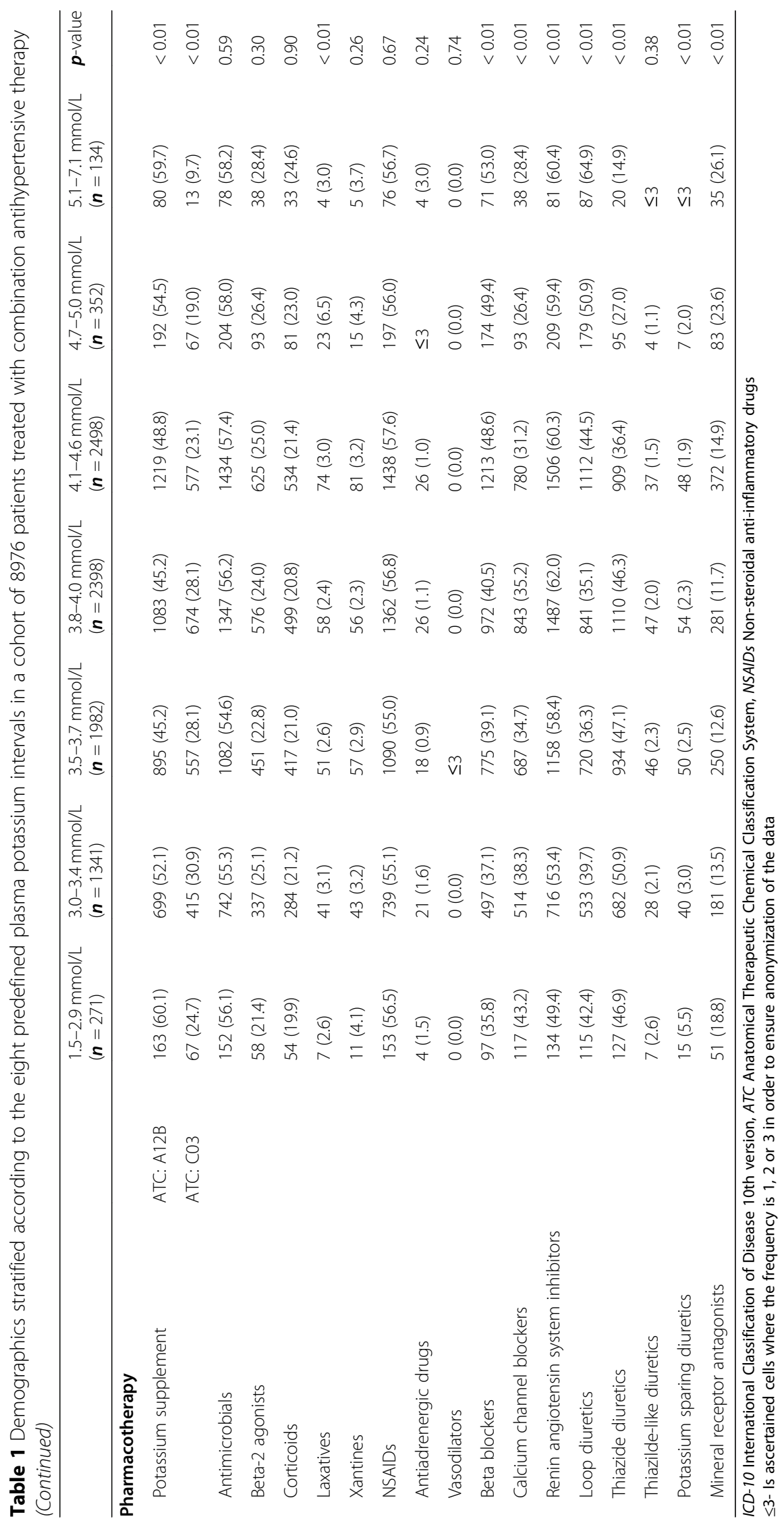




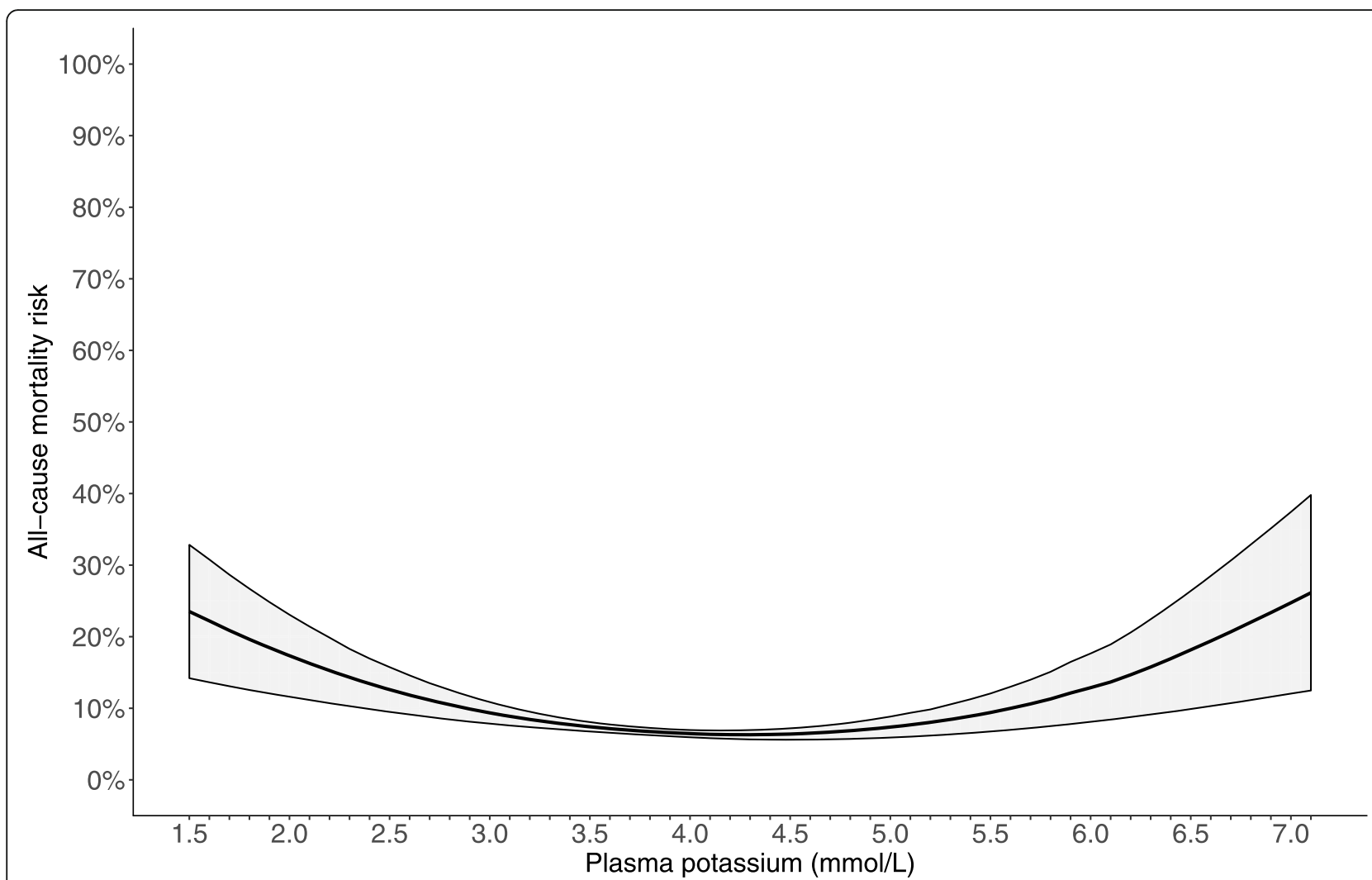

Fig. 1 Age, sex, comorbidity and drug standardized 60-day risk of all-cause death in relation to plasma potassium as a continuous variable. Model adjusted for age, gender, plasma sodium, renal insufficiency, malignancy, heart failure, chronic liver disease, chronic obstructive pulmonary disease, diabetes mellitus, atrial flutter/fibrillation, stroke and ischemic heart disease, antihypertensive therapy, corticosteroids, antimicrobials, nonsteroidal anti-inflammatory drugs, potassium supplement, xanthines, laxatives

instead of the first measurement, we noted that potassium levels below $3.8 \mathrm{mmol} / \mathrm{L}$ were associated with increased short-term mortality.

Eighth, analyses on patients with available $K_{2}$ measurements within 6-45 days from $K_{1}$, showed that severe hypokalemia and hyperkalemia were associated with 60day all-cause mortality.

Ninth, analyses on patients with available $K_{2}$ measurements above 45 days from $K_{1}$, showed that potassium interval $3.0-3.4 \mathrm{mmol} / \mathrm{L}$ was associated with 60 -day allcause mortality.

Tenth, we stratified $K_{2}$ in three intervals: 1.5-3.4 $\mathrm{mmol} / \mathrm{L}$ (hypokalemia), 3.5-4.6 mmol/L (normokalemia) and 4.7-7.1 mmol/L (hyperkalemia). Mortality within 60-days was increased both in patients with hypokalemia (HR 1.36, 95\% CI 1.12-1.66) and in patients with hyperkalemia (HR 2.13, 95\% CI 1.66-2.74) at $\mathrm{K}_{2}$ measurement compared with patients with normal potassium concentrations.

Eleventh, multivariable analysis on patients with available magnesium measurements at the time of plasma potassium draws, showed significant association of potassium levels below $3.0 \mathrm{mmol} / \mathrm{L}$ and mortality (HR 2.46,
95\% CI 1.05-5.74). In addition, we also observed a trend towards increased risk of death in patients with potassium between 3.0-3.4 mmol/L.

\section{Discussion}

This Danish register-based cohort study investigated 60day mortality among 8976 patients with hypertension and hypokalemia or low normal potassium in relation to a subsequent potassium measurement. The major findings were: (1) Persistent hypokalemia following low potassium was more than twice as frequent as development of hyperkalemia. (2) Persistent hypokalemia was common and associated with increased all-cause and presumed cardiovascular mortality; (3) Increase in potassium to levels $>4.6 \mathrm{mmol} / \mathrm{L}$ in patients with initial hypokalemia or low normal potassium was associated with increased all-cause and cardiovascular mortality; (4) Among patients with borderline hypokalemia initially, development of hypokalemia or hyperkalemia was associated with increased mortality risk; (5) Correcting hypokalemia associated with increased survival.

In the current study, we observed significantly higher 60-day mortality risk in patients with potassium 


\section{All-cause mortality}

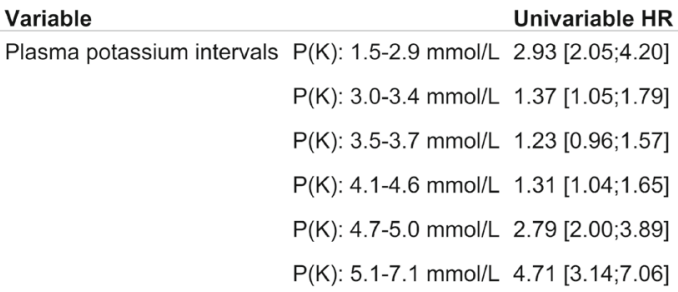

Multivariable $\mathrm{HR}[95 \% \mathrm{Cl}]$

$2.39[1.66,3.43]$

$1.36[1.04,1.78]$

$1.24[0.97,1.59]$

$1.20[0.95,1.51]$

$2.36[1.68,3.30]$

$2.62[1.73,3.98]$

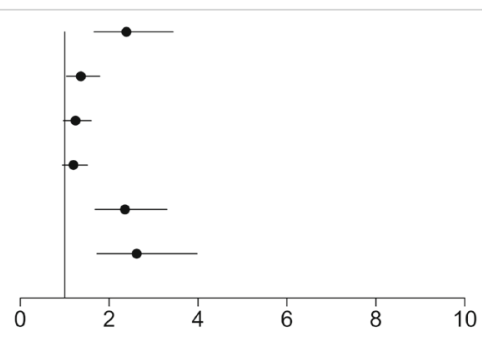

\section{Cardiovascular mortality}

\begin{tabular}{|c|c|c|}
\hline Variable & Univariable HR $[95 \% \mathrm{Cl}]$ & Multivariable HR $[95 \% \mathrm{Cl}]$ \\
\hline \multirow[t]{6}{*}{ Plasma potassium intervals } & $\mathrm{P}(\mathrm{K}): 1.5-2.9 \mathrm{mmol} / \mathrm{L} \quad 2.57[1.53 ; 4.34]$ & $2.32[1.36,3.93]$ \\
\hline & $\mathrm{P}(\mathrm{K}): 3.0-3.4 \mathrm{mmol} / \mathrm{L} \quad 1.22[0.83 ; 1.79]$ & $1.33[0.90,1.95]$ \\
\hline & $\mathrm{P}(\mathrm{K}): 3.5-3.7 \mathrm{mmol} / \mathrm{L} \quad 1.07[0.75 ; 1.52]$ & $1.11[0.77,1.58]$ \\
\hline & $\mathrm{P}(\mathrm{K}): 4.1-4.6 \mathrm{mmol} / \mathrm{L} \quad 1.15[0.82 ; 1.59]$ & $1.00[0.72,1.39]$ \\
\hline & $\mathrm{P}(\mathrm{K}): 4.7-5.0 \mathrm{mmol} / \mathrm{L} 2.21[1.34 ; 3.65]$ & $1.76[1.06,2.92]$ \\
\hline & $\mathrm{P}(\mathrm{K}): 5.1-7.1 \mathrm{mmol} / \mathrm{L} \quad 4.63[2.64 ; 8.11]$ & $2.64[1.48,4.71]$ \\
\hline
\end{tabular}

$\mathrm{P}(\mathrm{K}): 5.1-7.1 \mathrm{mmol} / \mathrm{L} 4.63[2.64 ; 8.11]$

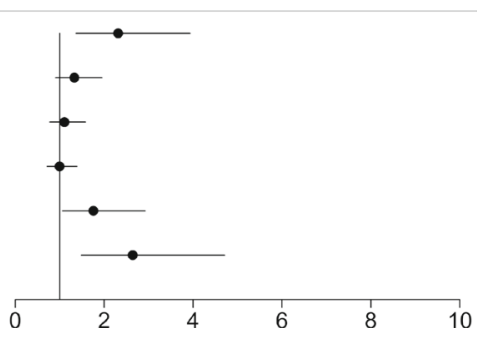

Fig. 2 All-cause and cardiovascular mortality after hypokalemia or borderline hypokalemia according to subsequent potassium measurements in patients treated with combination antihypertensive therapy (60-days follow-up, $n=8976$ ). Potassium interval K: $3.8-4.0$ mmol/L represented the reference range. Adjusted for age, gender, serum sodium, renal insufficiency, malignancy, heart failure, chronic liver disease, chronic obstructive pulmonary disease, diabetes mellitus, atrial flutter/fibrillation, stroke and ischemic heart disease, antihypertensive therapy, corticosteroids, antimicrobials, non-steroidal anti-inflammatory drugs, potassium supplement, xanthines, laxatives

concentrations $<3.5$ or $>4.6 \mathrm{mmol} / \mathrm{L}$ after an episode with hypokalemia or low normal potassium. This finding was not surprising as we previously observed an apparent optimal potassium range within $4.1-4.7 \mathrm{mmol} / \mathrm{L}$ in a similar population [5]. Of 8976 patients with initial plasma potassium $\leq 3.7 \mathrm{mmol} / \mathrm{L}, 18 \%$ had potassium concentrations $\leq 3.7 \mathrm{mmol} / \mathrm{L}$ at the second measurement and $5.4 \%>4.6 \mathrm{mmol} / \mathrm{L}$, suggesting that potassium deficit is frequently underestimated than overestimated by physicians. Notably, $13 \%$ of the patients with borderline hypokalemia (K: 3.5 and $3.7 \mathrm{mmol} / \mathrm{L}$ ) at the first measurement experienced a further decrease in potassium $(<3.5 \mathrm{mmol} / \mathrm{L})$ at the second measurement. This suggests that the association of low normal potassium

Table 2 60-day standardized absolute risk for all-cause death after hypokalemia or borderline hypokalemia according to subsequent potassium measurements in patients treated with combination antihypertensive therapy $(n=8976)$. Potassium interval K: 3.8-4.0 $\mathrm{mmol} / \mathrm{L}$ represented the reference range. Adjusted for age, gender, serum sodium, renal insufficiency, malignancy, heart failure, chronic liver disease, chronic obstructive pulmonary disease, diabetes mellitus, atrial flutter/fibrillation, stroke and ischemic heart disease, antihypertensive therapy, corticosteroids, antimicrobials, non-steroidal anti-inflammatory drugs, potassium supplement, xanthines, laxatives

\begin{tabular}{|c|c|c|c|c|c|}
\hline & Absolute risk \%, $(95 \% \mathrm{Cl})$ & 60-d Risk difference \%, (95\%Cl) & $p$-value & Average risk ratio \%, $(95 \% \mathrm{Cl})$ & $p$-value \\
\hline$P(K) 1.5-2.9 \mathrm{mmol} / \mathrm{L}$ & $11.7 \%(8.3-15.0)$ & $6.3(2.9-9.7)$ & $<0.001$ & $2.17(1.46-2.88)$ & 0.001 \\
\hline$P(K) 3.0-3.4 \mathrm{mmol} / \mathrm{L}$ & $7.1 \%(5.8-8.5)$ & $1.7(0.1-3.4)$ & 0.03 & $1.32(0.99-1.66)$ & 0.06 \\
\hline$P(K)$ 3.5-3.7 mmol/L & $6.4 \%(5.3-7.5)$ & $1.0(-0.3-2.4)$ & 0.14 & $1.19(0.91-1.47)$ & 0.17 \\
\hline$P(K) 3.8-4.0 \mathrm{mmol} / \mathrm{L}$ & $5.4 \%(4.5-6.3)$ & REF. & & REF. & \\
\hline$P(K) 4.1-4.6 \mathrm{mmol} / \mathrm{L}$ & $6.3 \%(5.4-7.2)$ & $0.9(-0.3-2.2)$ & 0.13 & $1.18(0.92-1.44)$ & 0.17 \\
\hline$P(K)$ 4.7-5.0 mmol/L & $11.6 \%(8.7-14.6)$ & $6.2(3.2-9.3)$ & $<0.001$ & $2.17(1.51-2.82)$ & $<0.001$ \\
\hline$P(K) 5.1-7.1 \mathrm{mmol} / \mathrm{L}$ & $12.6 \%(8.2-16.9)$ & $7.2(2.8-11.6)$ & 0.001 & $2.34(1.45-3.22)$ & 0.003 \\
\hline
\end{tabular}


concentrations with mortality that we previously observed [5] can partly be explained by further declines in potassium levels, and that low normal potassium concentrations might be a marker for an ongoing decrease in potassium.

Our results also suggest that correction of hypokalemia is important in relation to short-term mortality, as patients in the middle of the normal reference interval had good prognosis. Guidelines recommend supplementation with potassium when plasma potassium levels are below $3.5 \mathrm{mmol} / \mathrm{L}$. [13] However, in this study we cannot elucidate because of the low follow-up time the mechanism through which patients increased or decreased in potassium concentrations. It is also difficult to state whether potassium is a risk factor or a risk marker regarding mortality. Our population is relatively old, patients are treated with at least two antihypertensive drugs, and about $20 \%$ of the patients have history of heart failure, ischemic heart disease, atrial fibrillation/ flutter, chronic obstructive pulmonary disease and diabetes. Possibly, potassium concentrations at noncardiotoxic levels more likely are a risk marker of great disease burden, which is very important to recognize and identify.

Potassium supplementation of asymptomatic patients with low normal concentrations is controversial. Guidelines in the US recommend a stricter standard for potassium replacement therapy $(<4.0 \mathrm{mmol} / \mathrm{L})$ especially in patients with cardiovascular disease who are at high risk of ventricular tachyarrhythmias [13]. Our study suggests that potassium concentrations in the middle of the reference interval are beneficial even in patients with potassium levels $\leq 3.7 \mathrm{mmol} / \mathrm{L}$.

Various studies have previously demonstrated that hypokalemia among patients with cardiovascular disease is associated with an increased mortality risk [14-18]. However, no prior studies have investigated the impact of potassium normalization on short-term survival. Though, one study examined the impact of correcting hypokalemia within $24 \mathrm{~h}$ on the risk of cardiac arrhythmias in hospitalized patients without coronary syndromes or history of arrhythmias [19]. The authors did not find increased odds of arrhythmia in patients with hypokalemia whose potassium levels were not corrected $\geq 3.5 \mathrm{mmol} / \mathrm{L}$. Although, the study does not describe or account for the cause of admission, comorbidities or pharmacotherapy. The investigators excluded patients with history of ischemic heart disease and arrhythmia, but included patients with heart failure who have a high arrhythmia risk. Overall, both the study population and the outcome measure differed in this paper compared with our study.

Another study performed on 5916 individuals from the general population found no significant associations between borderline hypokalemia $(3.4-3.6 \mathrm{mmol} / \mathrm{L})$ and risk of all-cause mortality, risk of stroke or risk of acute myocardial infarction [20]. Comparing the results of our study with this study is difficult due to major differences in study population, methodology and aim. First, our population was characterized by redemption of at least two antihypertensive drugs. Mattsson et al. [20] enrolled participants from the general population, where $49.6 \%$ had high blood pressure at baseline, $13.9 \%$ were prescribed heart medication and $10.9 \%$ were treated with diuretics. In our population, we observed higher burden of cardiovascular disease and use of diuretics. Second, our aim was to investigate the impact of correcting hypokalemia or borderline hypokalemia on short-term all-cause and cardiovascular mortality. In terms of mortality, Mattsson et al. [20] followed participants from their fourth examination in 2001-2003 until November 2014 or death, having a median follow-up of 11.9 years (Q1-Q3: 11.4-12.5 years). As potassium is varying over time especially in patients with cardiovascular disease or treated with antihypertensive drugs, use of one potassium measurement to assess mortality over more than 10 years can provide results that are difficult to interpret. Shorter follow-up time or time varying analysis where the authors accounted for both multiple measurements over time and change in relevant medication would have provided better methodology. Although, it is important to acknowledge that correcting hypokalemia and low normal potassium might not have the same impact in general population compared to a population with heart disease.

Another study investigated the influence of dyskalemia at admission and early dyskalemia correcting on shortterm survival and cardiac events among intensive care unit (ICU) patients [21]. The authors concluded that patients with persisting hypokalemia or hyperkalemia within the first 2 days in ICU had increased risk of death. The two populations are not comparable, however both studies emphasize the importance of rapid correction of hypokalemia to improve short-term mortality.

\section{Limitations}

The limitations are related to the observational nature of register-based studies, which imply non-causal interpretation of the results.

We did not have information about comorbidities and risk factors from the primary sector. Therefore, patients who did not redeem any medication of interest or were not registered an ICD-code from the secondary sector could have been misclassified as "healthy". Patients with complications related to hypertension have a larger likelihood for being referred to the secondary sector and therefore also a higher probability for being diagnosed with other conditions (compared with patients with 
uncomplicated hypertension), leading to an ascertainment/surveillance bias and non-differential misclassification bias. To reduce this bias, we defined hypertension as use of at least two antihypertensive drugs in two concomitant quarters. Whether hypertension was resistant, controlled or uncontrolled was unkown, and data about ejection fraction and type of heart failure was not available.

We cannot exclude that the blood draws may contain hemolysis. However, in case of significant hemolysis the samples submitted are rejected and no potassium value is available.

We could not investigate the effect of any potential treatment or drug dosage adjustment in the time between the first and second potassium measurement. The Danish National Prescription Registry records filled prescriptions; thus, changes in dosage cannot be identified, unless a new drug is prescribed. In addition, the majority of the patients were hospitalized at the time of potassium measurement and any treatment during hospitalization is not registered in the Danish National Prescription Registry. Moreover, it was also difficult to identify the cause of hypokalemia using the registers. Hypokalemia might have occurred due to administration of diuretics, alkalosis, derangements in the renin angiotensin aldosterone system, gastroenteritis or other pathologies. However, the purpose of this study was neither to investigate the cause of hypokalemia, nor to assess the strategies used to correct low potassium concentrations. The purpose of this study was to find a clue, whether normalization of potassium had an effect on short-term mortality, whether we should increase potassium concentrations in patients with borderline hypokalemia and whether potassium actually increased.

It is also important to acknowledge that plasma potassium is not always a good predictor of the whole body potassium. Yet, it is the most commonly used method to assess potassium and only in patients with persistent hypokalemia over a longer period of time total body potassium is calculated.

\section{Conclusion}

Persistent hypokalemia was frequent and associated with increased all-cause and cardiovascular mortality. Increase in potassium to levels $>4.6 \mathrm{mmol} / \mathrm{L}$ in patients with initial hypokalemia or low normal potassium was associated with increased all-cause and cardiovascular mortality.

\section{Perspectives}

We were not able to report the initiatives medical doctors undertook after observing potassium levels below $3.8 \mathrm{mmol} / \mathrm{L}$ at the first measurement. However, our results emphasize the importance of potassium normalization after an episode with hypokalemia and low normal potassium and that overcorrection is associated with an increased risk of death. Potassium concentrations in the middle of the normal reference interval are associated with good prognosis. Possibly, potassium supplementation, use of mineral receptor antagonists or thiazide-like diuretics instead of thiazide-type in patients with potassium concentrations $\leq 3.7 \mathrm{mmol} / \mathrm{L}$ could be of clinical importance, but requires further study, preferably through a randomized controlled trial.

\section{Supplementary information}

Supplementary information accompanies this paper at https://doi.org/10. 1186/s12872-020-01654-3.

\section{Additional file 1.}

\section{Abbreviations}

ATC: Anatomical therapeutic chemical classification system; $\mathrm{K}_{1}$ : First potassium measurement within 100 days from combination antihypertensive therapy initiation; $\mathrm{K}_{2}$ : First potassium measurement within 6-100 days from $\mathrm{K}_{1}$; ICD: International classification of disease; CKD-EPI: Chronic kidney disease epidemiology collaboration; eGFR: Estimated glomerular filtration rate; HR: Hazard ratio; AR: Absolute risk; 95\% Cl: 95\% confidence interval; NSAI Ds: Non-steroidal anti-inflammatory drugs

\section{Acknowledgements}

None.

\section{Authors' contributions}

Conception or design of the work: MLK, PS, CTP, KK, JQT, AB, HB. Acquisition of data: MLK, PS, CTP, KK, AB, CJYL. Analysis and interpretation of data: MLK, $P S, C T P, A B, K K, C J Y L, H B$, JQT. Draftet the manuscript: MLK. Critically revised the manuscript: Peter Søgaard, Christian Torp-Pedersen, Henrik Bøggild, Christina Ji-Young Lee, Anders Bonde, Jesper Q. Thomassen, Gunnar Gislason, Manan Pareek, Kristian Kragholm, All authors gave final approval and agree to be accountable for all aspects of work ensuring integrity and accuracy.

\section{Funding}

This study was funded using departmental funding sources only. The funding covered labor costs.

\section{Availability of data and materials}

Due to restrictions related to Danish law and protecting patient privacy, the combined set of data used in this study can only be made available through a trusted third party, Statistics Denmark. This state organisation holds the data used for this study. University-based Danish scientific organisations can be authorized to work with data within Statistics Denmark and such organisation can provide access to individual scientists inside and outside of Denmark. Data are available upon request to authorized scientists by contacting Statistics Denmark: http://www.dst.dk/en/OmDS/organisation/TelefonbogOrg.aspx?kontor=13\&tlfbogsort=sektion or the Danish Data Protection Agency: https://www.datatilsynet.dk/english/the-danish-data-protectionagency/contact/. More information regarding data access is available at https://www.dst.dk/en/TilSalg/Forskningsservice.

Ethics approval and consent to participate

Retrospective studies do not require ethics approval in Denmark and all data were deidentified and only available through Statistics Denmark. Approval from the Danish Data Protection Agency was secured, and the need for patient informed consent was not needed.

Consent for publication

Not applicable. 


\section{Competing interests}

None to declare.

\begin{abstract}
Author details
'Department of Cardiology, Aalborg University Hospital, Aalborg, Denmark. 2Department of Cardiology and Clinical Research, Nordsjællands Hospital, Hillerød, Denmark. ${ }^{3}$ Public Health and Epidemiology Group, Department of Health Science and Technology, Aalborg University, Aalborg, Denmark. ${ }^{4}$ Unit of Epidemiology and Biostatistics, Aalborg University Hospital, Aalborg, Denmark. ${ }^{5}$ Department of Cardiology, Copenhagen University Hospital, Herlev and Gentofte, Hellerup, Denmark. ${ }^{6}$ Department of Clinical Biochemistry, Rigshospitalet, University of Copenhagen, Copenhagen, Denmark. ${ }^{7}$ Department of Cardiology, Herlev and Gentofte University Hospital, Hellerup, Denmark. ${ }^{8}$ The Danish Heart Foundation, Copenhagen, Denmark. ${ }^{9}$ The National Institute of Public Health, University of Southern Denmark, Copenhagen, Denmark. ${ }^{10}$ Department of Internal Medicine, Yale New Haven Hospital, Yale University School of Medicine, New Haven, USA. ${ }^{11}$ Brigham and Women's Hospital, Heart \& Vascular Center, Harvard Medical School, Boston, USA. ${ }^{12}$ Department of Cardiology, Regionshospital Nordjylland, Hjørring, Denmark.
\end{abstract}

Received: 16 April 2020 Accepted: 4 August 2020

Published online: 24 August 2020

\section{References}

1. Veltri KT, Mason C. Medication-induced hypokalemia. P T United States. 2015;40:185-90.

2. Sica DA, Carter B, Cushman W, Hamm L. Thiazide and loop diuretics. J Clin Hypertens (Greenwich) United States. 2011;13:639-43.

3. Tamargo J, Segura J, Ruilope LM. Diuretics in the treatment of hypertension. Part 2: loop diuretics and potassium-sparing agents. Expert Opin Pharmacother. 2014;15(5):605-21.

4. Rodenburg EM, Visser LE, Hoorn EJ, Ruiter R, Lous JJ, Hofman A, Uitterlinden AG, Stricker BH. Thiazides and the risk of hypokalemia in the general population. J Hypertens. 2014;32:2092-7.

5. Krogager ML, Torp-Pedersen C, Mortensen RN, Køber L, Gislason G, Søgaard $P$, Aasbjerg K. Short-term mortality risk of serum potassium levels in hypertension: a retrospective analysis of nationwide registry data. Eur Heart J. 2017;38:104-12

6. Thygesen LC, Daasnes C, Thaulow I, Bronnum-Hansen H. Introduction to Danish (nationwide) registers on health and social issues: structure, access, legislation, and archiving. Scand J Public Health Sweden. 2011;39:12-6.

7. Pedersen CB. The Danish civil registration system. Scand J Public Health. 2011;39:22-5.

8. Olesen JB, Lip GY, Hansen ML, Hansen PR, Tolstrup JS, Lindhardsen J, Selmer C, Ahlehoff O, Olsen AM, Gislason GH, Torp-Pedersen C. Validation of risk stratification schemes for predicting stroke and thromboembolism in patients with atrial fibrillation: nationwide cohort study. BMJ. 2011;342:d124 Department of Cardiology, Copenhagen University Hospital Gentofte, 2900 Hellerup, Denmark. jo@heart.dk.

9. Levey AS, Stevens LA, Schmid CH, Zhang Y, Castro AF, Feldman HI, Kusek JW, Eggers P, Van Lente F, Greene T, Coresh J. A new equation to estimate glomerular filtration rate. Ann Intern Med. 2009;150:604-12.

10. Burtis CA, Ashwood ER, Bruns DE. Tietz textbook of clinical chemistry and molecular diagnostics. 5th ed. St. Louis, Missouri, USA: Elsevier Saunders; 2012.

11. Drogies T, Ittermann T, Lüdemann J, Klinke D, Kohlmann T, Lubenow N, Greinacher A, Völzke H, Nauck M. Potassium - reference intervals for lithiumheparin plasma and serum from a population-based cohort. LaboratoriumsMedizin. 2010;34:39-44.

12. Article citationsMore $>>R$ Core Team. R: a language and environment for statistical computing. Vienna: R Foundation for Statistical Computing; 2018. https://www.R-project.org.

13. Cohn JN, Kowey PR, Whelton PK, Prisant LM. New guidelines for potassium replacement in clinical practice: a contemporary review by the National Council on potassium in clinical practice. Arch Intern Med United States. 2000;160:2429-36

14. Alderman MH, Piller LB, Ford CE, Probstfield $J$, Oparil S, Cushman WC, Einhorn PT, Franklin SS, Papademetriou V, Ong ST, Eckfeldt JH, Furberg CD, Calhoun DA, Davis BR, Group A and L-LT to PHATCR. Clinical significance of incident hypokalemia and hyperkalemia in treated hypertensive patients in the antihypertensive and lipid-lowering treatment to prevent heart attack trial. Hypertension. 2012;59:926-33 Department of Epidemiology and Social Medicine, Albert Einstein College of Medicine, Bronx, NY, USA.

15. Aldahl M, Jensen A-SC, Davidsen L, Eriksen MA, Moller Hansen S, Nielsen BJ, Krogager ML, Kober L, Torp-Pedersen C, Sogaard P. Associations of serum potassium levels with mortality in chronic heart failure patients. Eur Heart J England. 2017;38:2890-6.

16. Krogager ML, Eggers-Kaas L, Aasbjerg K, Mortensen RN, Køber L, Gislason G, Torp-Pedersen C, Søgaard P. Short-term mortality risk of serum potassium levels in acute heart failure following myocardial infarction. Eur Hear J Cardiovasc Pharmacother. 2015;1:245-51.

17. Hagengaard L, Søgaard $P$, Espersen M, et al. Association between serum potassium levels and short-term mortality in patients with atrial fibrillationor flutter co-treated with diuretics and rate- or rhythm-controlling drugs. Eur Heart J Cardiovasc Pharmacother. 2020;6(3):137-44.

18. Tishler M, Armon S. Nifedipine-induced hypokalemia. Drug Intell Clin Pharm United States. 1986;20:370-1.

19. Harkness W, Watts P, Kopstein M, Dziadkowiec O, Hicks G, Scherbak D. Correcting hypokalemia in hospitalized patients does not decrease risk of cardiac arrhythmias. Adv Med. 2019;2019:1-4.

20. Mattsson N, Nielsen OW, Johnson L, Prescott E, Schnohr P, Jensen GB, Kober $L$, Sajadieh A. Prognostic impact of mild hypokalemia in terms of death and stroke in the general population-a prospective population study. Am J Med United States. 2018;131:318.e9-318.e19.

21. Bouadma L, Mankikian S, Darmon M, Argaud L, Vinclair C, Siami S, Garrouste-Orgeas M, Papazian L, Cohen Y, Marcotte G, Styfalova L, Reignier J, Lautrette A, Schwebel C, Timsit JF, Timsit JF, Azoulay E, Garrouste-Orgeas M, Zahar JR, Adrie C, Darmon M, Clec'h C, Alberti C, Francąis A, Vesin A, Ruckly S, Bailly S, Lecorre F, Nakache D, Vannieuwenhuyze A, et al. Influence of dyskalemia at admission and early dyskalemia correction on survival and cardiac events of critically ill patients. Crit Care. 2019.

\section{Publisher's Note}

Springer Nature remains neutral with regard to jurisdictional claims in published maps and institutional affiliations.

Ready to submit your research? Choose BMC and benefit from:

- fast, convenient online submission

- thorough peer review by experienced researchers in your field

- rapid publication on acceptance

- support for research data, including large and complex data types

- gold Open Access which fosters wider collaboration and increased citations

- maximum visibility for your research: over $100 \mathrm{M}$ website views per year

At BMC, research is always in progress.

Learn more biomedcentral.com/submissions 